\title{
Ontario Association of Gastroenterology OHIP Gastroenterology Fee Guide October 2003*
}

*The fee guide is derived from the Ontario Schedule of Benefits for Physician Services (OHIP fee guide) which should be used as the definitive reference. At the meeting of the regional representation committee, the OAG fee guide was thought to be the most comprehensive and is being published as an illustration for a Canada-wide fee schedule. The regional representation welcomes and encourages submissions to it through the CAG from all jurisdictions to make it more comprehensive, so that a national schedule of procedures and visits can be created. We realize that there may be fee differences across the country, and that issue is separate from what should be available to patients in a universal health care system across the country. Please forward all feedback and suggestions to the committee via the CAG.

\section{ASSESSMENT FEES DEFINED}

Consultation

- Must be requested by a physician, but not a resident or intern.

- Request must be in writing if an outpatient.

- A consultation consists of a general or specific assessment (see below) with a written response to the referring physician.

- Each patient is eligible for a maximum of two consultations per calendar year (January 1 to December 31) as long as they are made as two separate requests for consultation and deal with clearly unrelated diagnoses.

- Any arrangement to see the patient back by the consultant no matter how much time has elapsed is not considered to have satisfied the criteria for consultation.

- There is no specific time component to a consultation as long as the criteria for a general or specific assessment is fulfilled.

\section{Repeat Consultation}

- Requires all the elements of a consultation including a request by the referring doctor in writing.

- There has been interval care by the primary physician.

- This is not a follow-up appointment for a previous consultation.

- This code may be used for a second consultation for same diagnosis during the same calendar year.

\section{Limited Consultation}

- This is a poorly defined code.

- Requires all the components of a consultation but is less demanding.

- In terms of time this consultation requires substantially less (not defined) of the physician's time than a full consultation.

\section{Medical Specific Assessment}

- Service rendered by a specialist.

- Requires a full history of presenting complaint and detailed examination of the affected part(s), region(s), or system(s) needed to make a diagnosis, and/or exclude disease and/or assess function.
- A patient is eligible for one specific assessment per 12 month period (not calendar year) unless:

i) the patient presents a second time within a 12 month period with a complaint which is clearly a different diagnosis is made, unrelated to the diagnosis made during the previous specific assessment; or

ii) at least 90 days have passed since the date of the last specific assessment and the service provided is a hospital in-patient admission assessment.

\section{Medical Specific Reassessment}

- Requires a full relevant history and comprehensive examination of one or more systems.

- Is to be used as the admission assessment by a specialist for an independent operative procedure (such as endoscopy) when the patient has been assessed by same specialist prior to admission for the same illness.

- Shall be billed as the admission assessment when those patients assessed by a specialist are subsequently admitted to hospital by the same physician for the same illness.

- a patient is eligible for two specific reassessments per 12 month period not including a specific reassessment used as an admission assessment.

\section{Complex Medical Specific Reassessment}

- Payable for reassessment of a patient because of the complexity, obscurity or seriousness of the patients condition.

- Must contain the elements of a medical specific reassessment.

- Consultant must make written report to primary physician.

- maximum 4 per patient per 12 month period

NOTE: Any combination of medical specific assessments and complex medical specific reassessments may not exceed four per patient per 12 -month period, ie, one may only bill three complex medical reassessments if one bills one medical specific assessment in any 12 -month period.

\section{Partial Assessment}

- Requires a history of the presenting complaint and appropriate physical examination.

- It is the service rendered for the purpose of subsequent visits for assessing the response to treatment and/or advice provided in a previous service. 


\section{GENERAL LISTINGS}

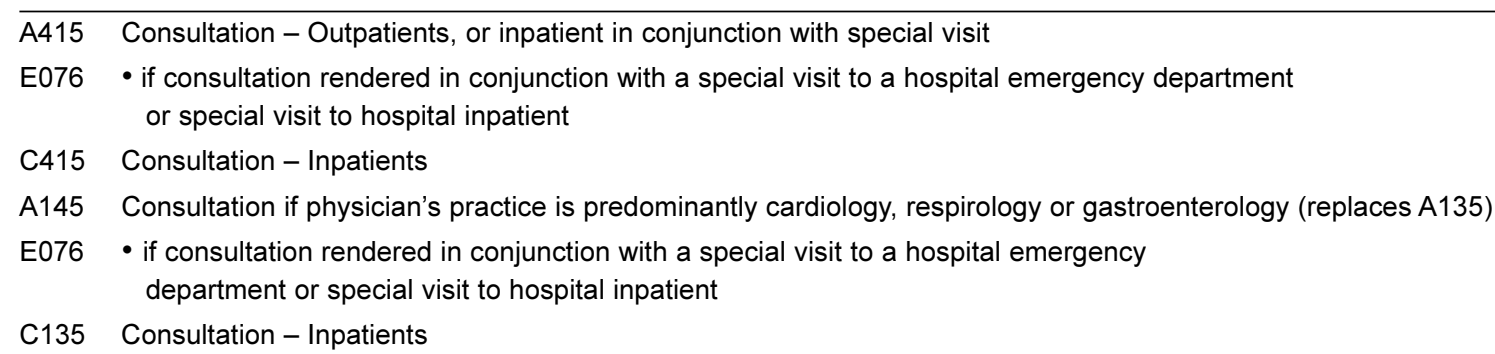

\section{A or C}

\begin{tabular}{ll}
\hline 545 & Limited Consultation - Gastroenterology \\
435 & Limited Consultation - Internal Medicine \\
416 & Repeat Consultation - Gastroenterology \\
136 & Repeat Consultation - Internal Medicine \\
413 & Medical Specific Assessment - Gastroenterology \\
133 & Medical Specific Assessment - Internal Medicine \\
414 & Medical Specific Re-assessment - Gastroenterology \\
134 & Medical Specific Re-assessment - Internal Medicine \\
411 & Complex medical specific Assessment - Gastroenterology \\
131 & Complex medical specific Assessment - Internal Medicine \\
A418 & Partial Assessment - Gastroenterology \\
A138 & Partial Assessment - Internal Medicine
\end{tabular}

\section{Subsequent Hospital Visits}

\begin{tabular}{ll}
\hline C412 & First five weeks, most responsible physician \\
C132 & \\
C417 & Weeks 6 to 13 inclusive, maximum 3 per patient per week \\
C137 & \\
C419 After 13 weeks, maximum 6 per month \\
C417 \\
C418 Concurrent care
\end{tabular}

C138

C121 Visits in excess of maximum allowed after 6 weeks, for intercurrent illness. No special documentation required at time of billing

\section{Special Visit Premiums}

All special visit fees are billed with an "A" prefix assessment codes, whether the patient is seen in an outpatient (ER, daycare) or as an inpatient.

Special visit codes do not apply to routine rounds, elective consultations, or when the physician travels from one location to another within the same hospital.

x990/x991 First/subsequent patient seen in ER (K), hospital ward (C), outpatient department (U) 0700-1800, 30\% premium.

x992/x993 First/subsequent patient seen with sacrifice of office hours. Had to leave office, cancel patients and have kept records of patients booked and cancelled $0700-1800,30 \%$ premium.

x994/995 First/subsequent patient seen 1800-2400 weekdays, all day weekends or holidays, 50\% premium.

x996/x997 First/subsequent patient seen 2400-0700, 75\% premium.

E409 Surgical/endoscopic procedure performed $1700-2400$ weekdays, all day weekends or holidays, $50 \%$ increase.

E410 Surgical/endoscopic procedure performed 0000-0700, 75\% increase.

Note: The time intervals for special visit for assessment and special visit for procedures are slightly different.

\section{Other Special Notes}

From time to time OHIP will invoke rules, which are not in the schedule of fees.

When billing in combination a colonoscopy with both snare polypectomy and fulguration of polyps, physicians will be required to submit their written report of the procedure to satisfy OHIP's concern that the snared polyp was not also the fulgurated polyp. In your dictation, you should make it clear that there was more than one polyp and that they were dealt with differently. 


\section{ESOPHAGUS}

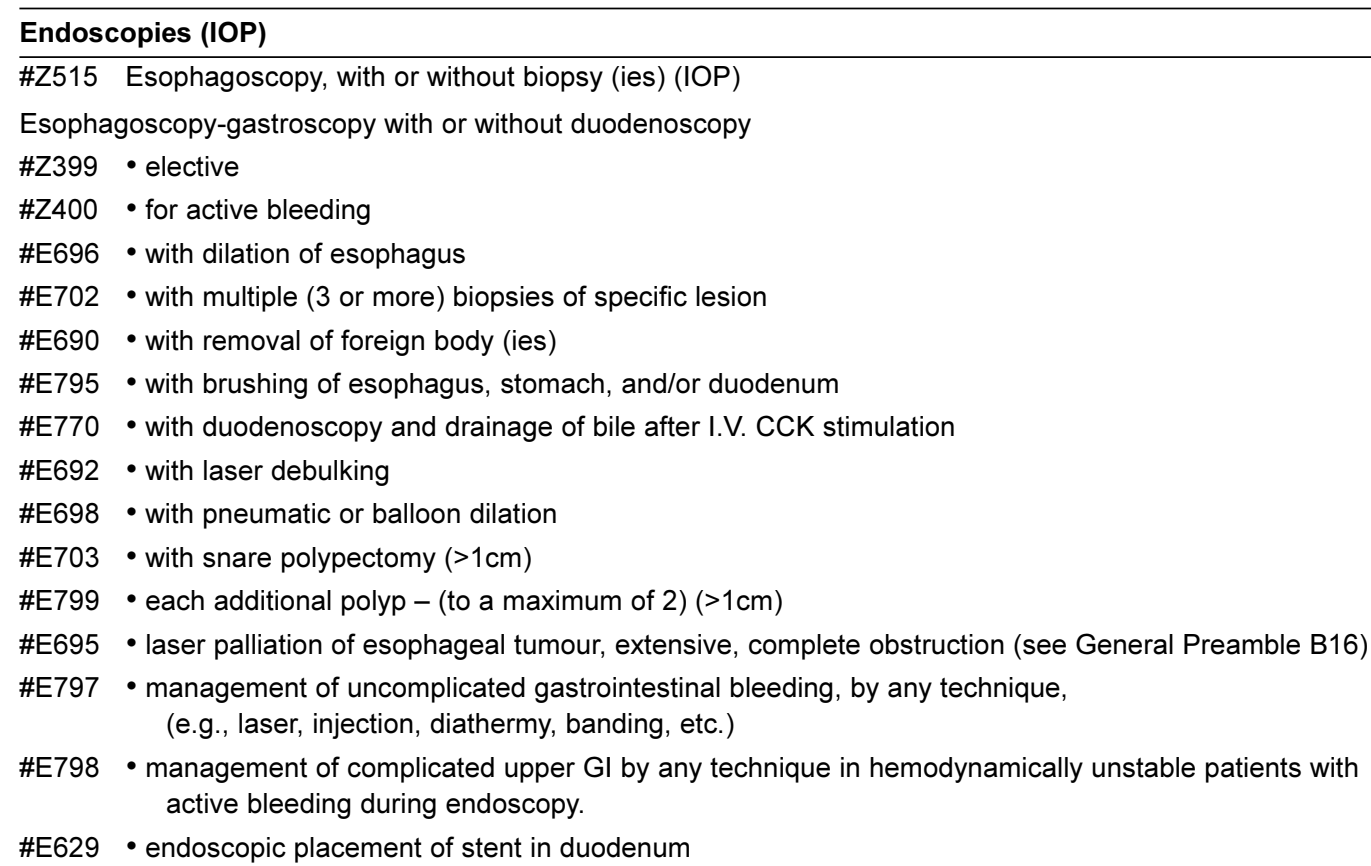

\section{Endoscopic Ultrasound}

\#E800 - Endoscopic ultrasound, radial or linear probe through endoscope, to endoscopy fee

Note: The amount payable for E800 when rendered in conjunction with E801 is zero

\#E801 - Endoscopic ultrasound, radial or linear probe through endoscope including biliary and/or pancreatic examination, to endoscope fee

Note: Amounts payable for upper and lower endoscopy rendered with S236 or S237 is zero unless the upper or lower $\mathrm{Gl}$ endoscopy is required due to the limited visualization with the linear or radial echo-endoscope

\#S236 - Endoscopic ultrasound using linear or radial echo-endoscope (scope also used for therapeutic procedures) excluding biliary or pancreatic examination. CANNOT BE BILLED WITH S237

\#S237 - Endoscopic ultrasound using linear or radial echo-endoscope (scope also used for therapeutic procedures) including biliary and/or pancreatic examination. CANNOT BE BILLED WITH S236

\#E802 - Biopsy or fine needle aspiration per lesion, to a maximum of 3 per lesion

\#E803 - Dilation of stricture

\#E804 - Injection of one or more of any of the following:metastases, nodes, masses, or celiac plexus

\#E805 - Drainage of pseudocyst (including stent insertion if performed)

Stenting of Esophagus

\#S083 • via esophagoscope (includes Z515)

\section{STOMACH}

\section{Endoscopies (IOP)}

\#Z527 Gastroscopy (may include biopsies, photography and removal of polyps $>1 \mathrm{~cm}$ )

\#Z547 Gastroscopy (with removal of foreign body) 


\section{CAG News}

\section{Stomach - continued}

\#Z528 Subsequent (within three months following previous gastroscopy)

\#E674 - with snare polypectomy - 1st polyp $>1 \mathrm{~cm}$ (maximum 1)

\#E675 each additional polyp $>1 \mathrm{~cm}$ (maximum 2)

Note: E674 and E675 are payable with Z527, Z547,Z547 or Z528

Incision

\#S119 Percutaneous endoscopic gastrostomy

Z520 Change of gastrostomy tube

INTESTINES (EXCEPT RECTUM)

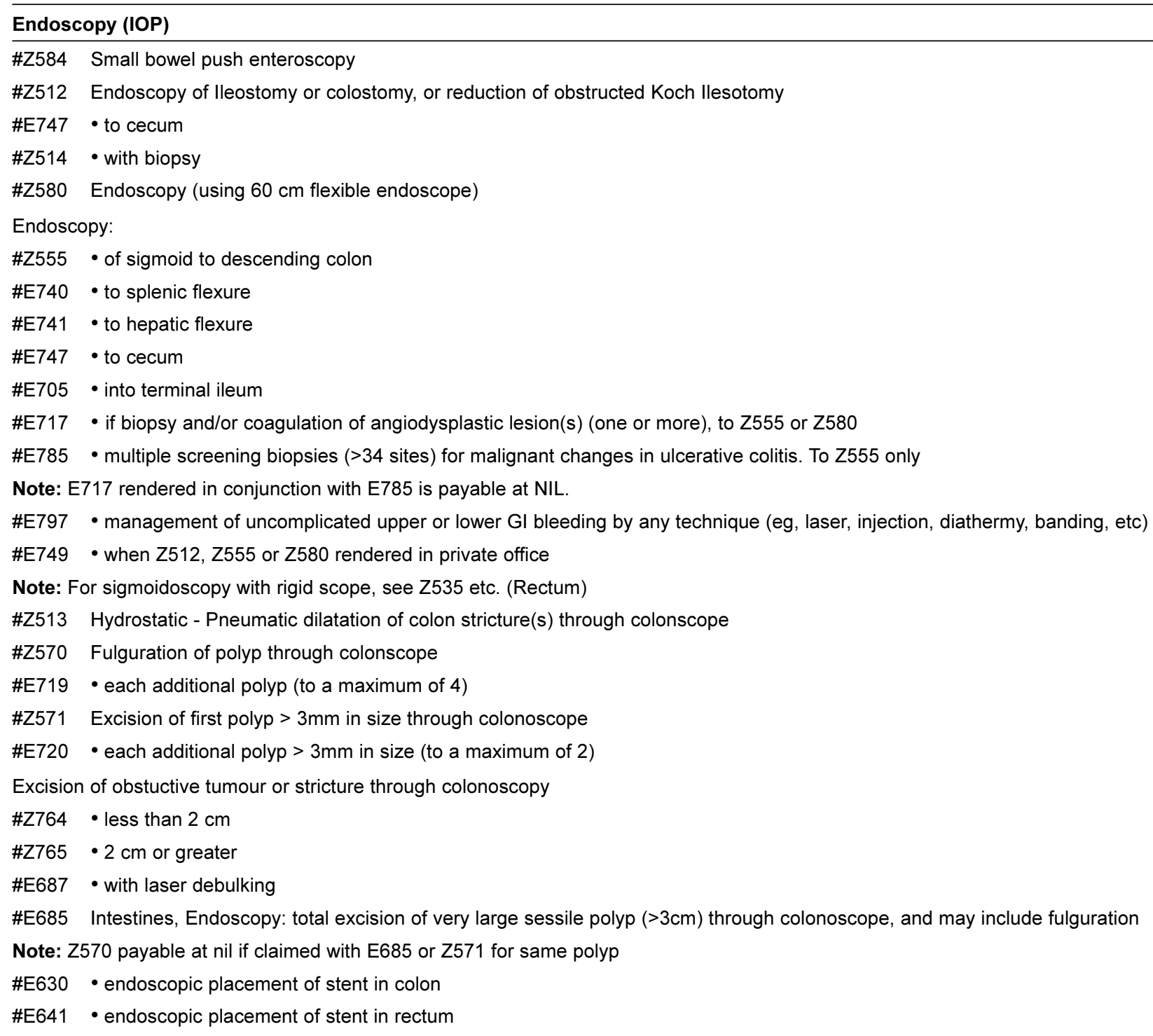

\section{RIGID SIGMOIDOSCOPY}

\begin{tabular}{ll}
\hline Sigmoidoscopy (with rigid scope) with or without anoscopy (IOP \\
\hline$\# Z 535$ & $\bullet$ not to be billed with Z555 or Z580 \\
$\# Z 536$ & $\cdot$ with biopsy(ies) \\
$\# Z 592$ & $\bullet$ with decompression of volvulus \\
$\# Z 746$ & $\bullet \cdot$ when Z535, Z536 or Z592, performed outside hospital
\end{tabular}




\section{RECTUM}

\section{Manipulation (IOP)}

\#Z541 Dilation and/or disimpaction or removal of foreign body under general anesthetic (when sole procedure performed)

\#Z756 Fecal disimpaction - no anaesthetic

Note: The fees for excision, ligation, injection or hemorrhoids and treatment of intra or perianal condylomata acuminata include anoscopy.

\section{Endoscopy}

\#Z543 Anoscopy (proctoscopy) (IOP)

\#E641 Endoscopic placement of stent in rectum

Incision

\#Z544 Biopsy (IOP)

\#Z545 Thrombosed hemorrhoid(s) (IOP)

\#E542 • when performed outside hospital

\#S241 Sphincterotomy(ies)

\#S242 • with excision of fissure(s)

\section{Excision}

\#S246 Excision of fissure(s)

\#S247 Hemorrhoidectomy, with or without sigmiodscopy or repair of fissure(s) and/or sphinctorotomy and/or anal dilation

\#Z565 Complete hemorrhoidectomy using cryotheraphy and/or Barron ligation(s) including rectal dilation (IOP)

\#Z546 Barron ligation(s) (IOP) (not to exceed 6 in any one year)

\#Z566 Barron ligation(s) plus cryotheraphy (IOP) (not to exceed 6 in any one year)

\#S249 Local excision for malignancy

Z757 Excision of benign anal lesion(s) (IOP)

E542 - when performed outside hospital

Injection

Z575 Hemorrhoid injections (IOP) (to a maximum of 6 per year)

Z576 Injections for anal fissure (IOP)

LIVER

Incision

\#Z554 Biopsy, incision (IOP)

\#Z551 Biopsy, needle (IOP)

\section{ABDOMEN, PERITONEUM AND OMENTUM}

Paracentesis (IOP)

\#Z590 Aspiration for diagnostic sample

\#Z591 Aspirations with therapeutic drainage with or without diagnostic sample

\#E724 - administration of chemotheraphy or sclerosing agent

\#Z763 Paracentesis with lavage for diagnosis (IOP)

\#E542 • when performed outside hospital (Z590, Z591, Z763)

BILIARY TRACT

Endoscopy (IOP)

Note: No extra fee for cholangiogram during abdominal surgery

\#Z561 Endoscopic retrograde cholangiopancreatography (ERCP) with cannulation of the common bile duct and/or pancreatic duct.

\#Z558 ERCP including sphincterotomy and may include removal of 1 or more bile duct stones

\#Z760 ERCP through gastrojejunostomy following previous Billroth II

\#E702 • with multiple (3 or more) biopsies of a specific lesion

\#E666 - with biliary tract monometry 


\section{CAG News}

\section{Biliary tract: Endoscopy (IOP) - continued}

\#E662 • with intraductal cytology brushing or intraductal biopsy

\#E668 • with cannulation of minor papilla

\#E680 • with insertion of first endobiliary prosthesis and/or pancreatic stent (maximum 1)

\#E681 - with insertion of each additional endobiliary prosthesis and/or pancreatic stent (maximum of 3 )

\#E669 - with esophagoscopy-gastroscopy may include duodenscopy

Note: E662, E666, E668, E702, E680, E681, E669, are payable with Z561, Z558 or Z760

\section{PANCREAS}

\section{Incision}

\#Z762 Biopsy, needle

\#Z577 Biopsy, incisional

\#S297 Drainage of acute pancreatitis or abscess or marsupialization of cyst

IOP Intraoperative procedure 


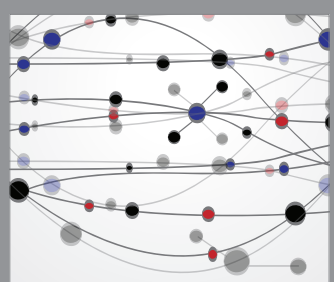

The Scientific World Journal
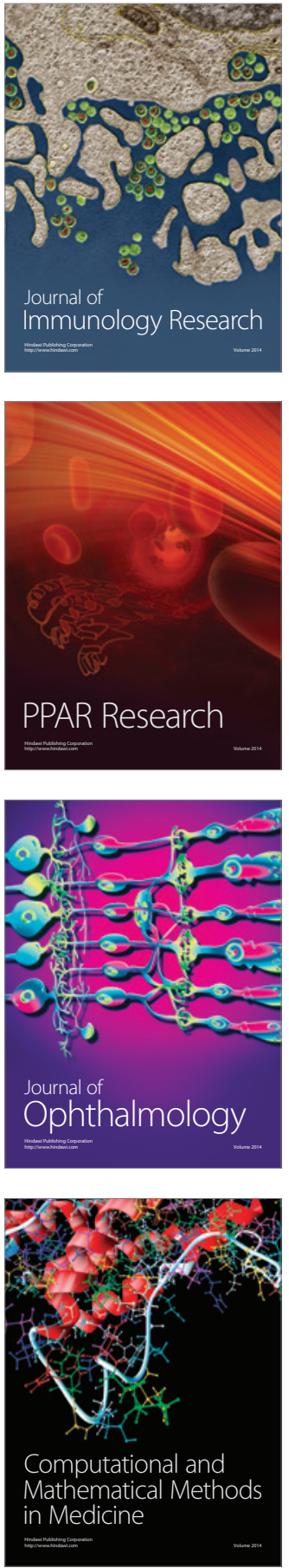

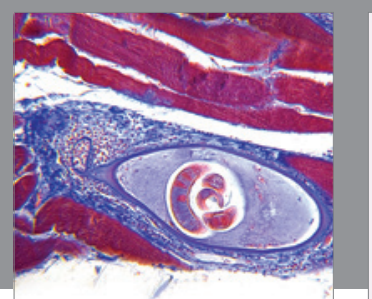

Gastroenterology Research and Practice

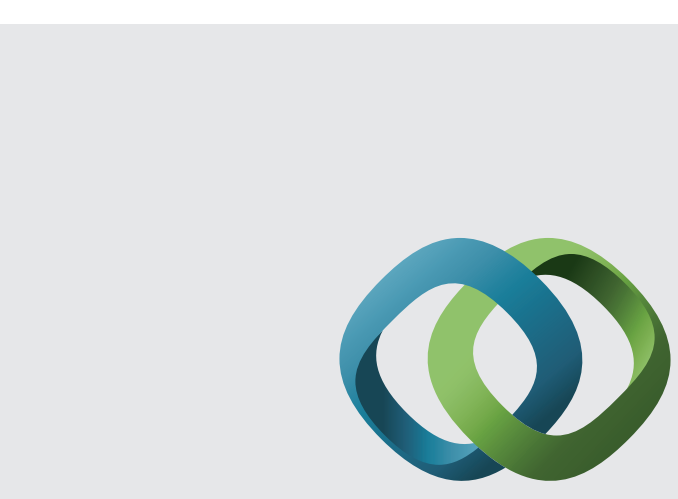

\section{Hindawi}

Submit your manuscripts at

http://www.hindawi.com
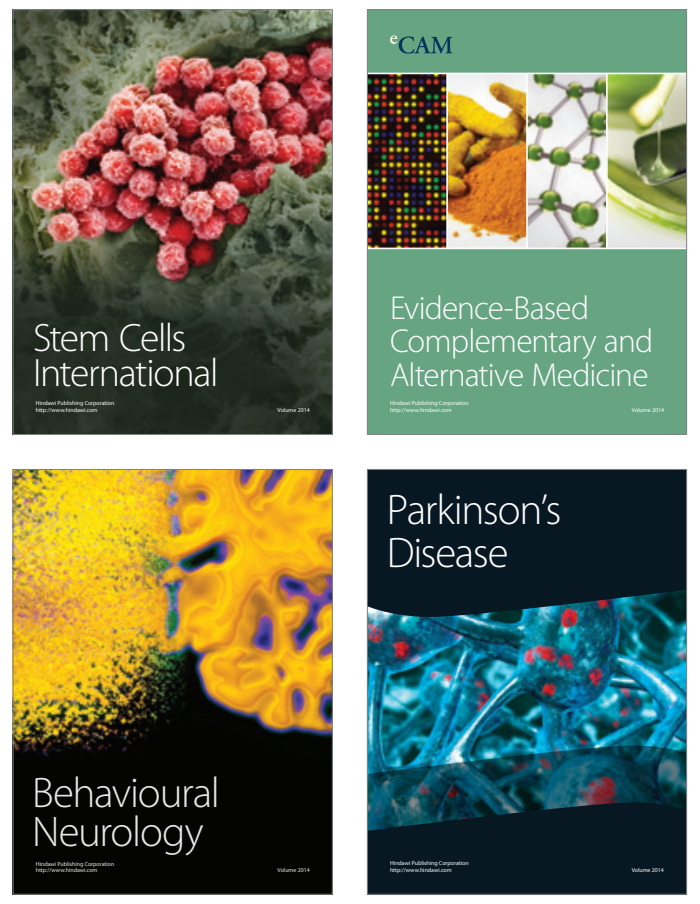
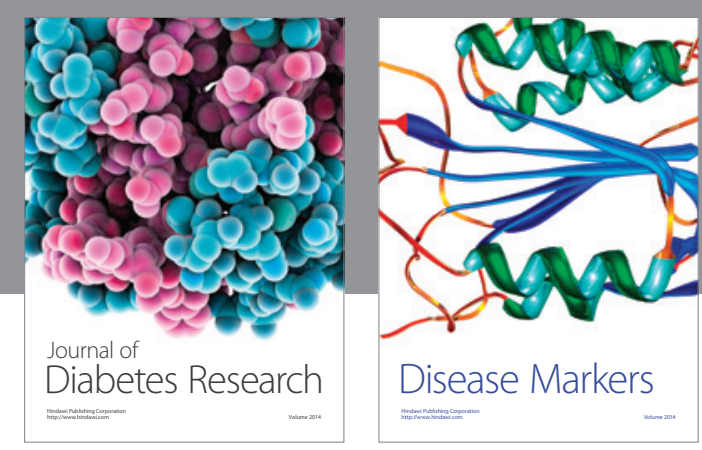

Disease Markers
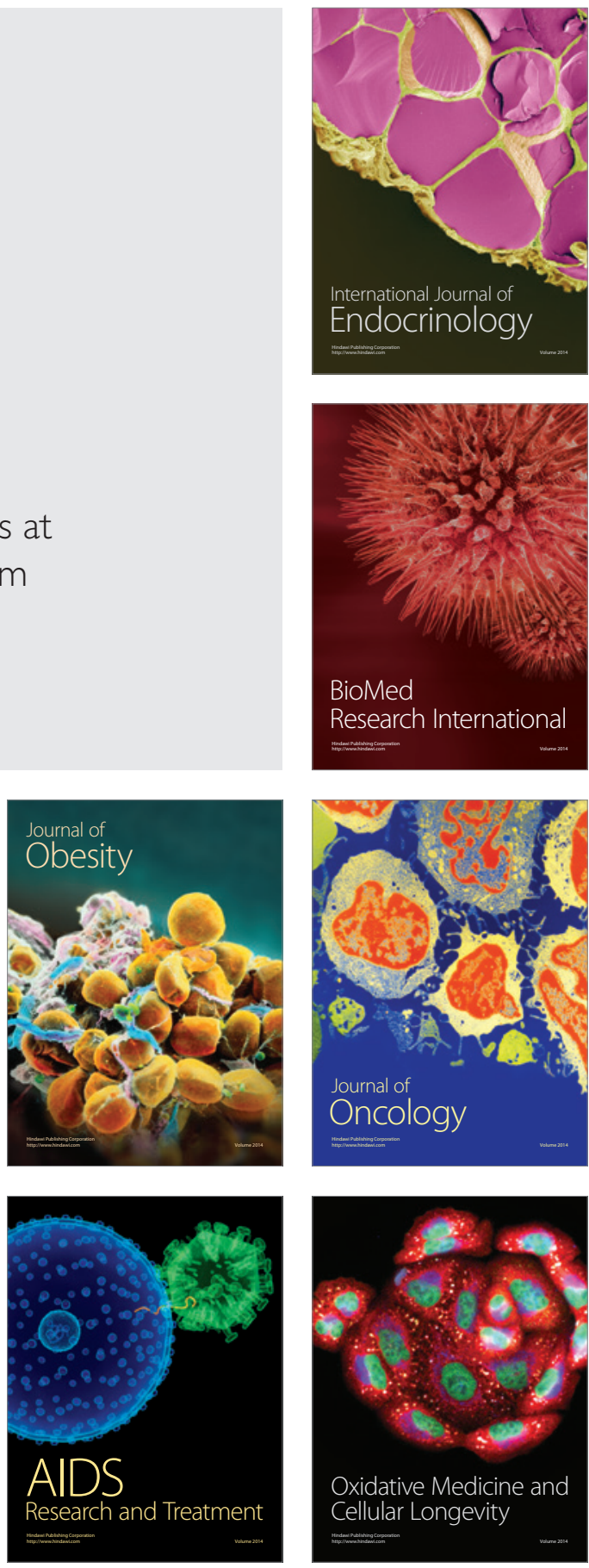